\title{
Healthcare in Singapore: the present and future
}

\author{
Choon How $\underline{H o w}^{1}$, MMed, FCFP, Kwong Ming $\underline{\text { Fock }^{2}}$, FAMS, FACP
}

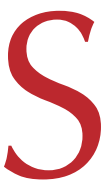

ingapore's rapidly evolving economy and social progress has helped accelerate the milestones of healthcare development in the country. ${ }^{(1)}$ In 1970, a committee on specialisation was commissioned by the Singapore government to look into the development of specialised medical services and postgraduate specialty training. Since then, there has been a steady focus on the development of our healthcare sector and the advancement of medical specialties. This has catapulted Singapore's health system to a level on par with other advanced nations. ${ }^{(2)}$

Today, healthcare systems around the world spend trillions of dollars annually to tackle the growing challenges in healthcare. ${ }^{(2-6)}$ These challenges include epidemiological transitions of death rates (from maternal, perinatal and infectious diseases to cardiovascular diseases and cancers) and an ageing population with improved life expectancies. ${ }^{(2,7)}$ Current lifestyle trends, which are by and large the result of technological advancements, have led to individuals having an increased number of risk factors, leading to the increased prevalence of multiple chronic diseases. ${ }^{(2,6)}$ These chronic diseases are also increasingly being diagnosed in the younger adult population in the recent years. ${ }^{(2,4-6,8)}$ Such trends will result in greater financial costs for the nation and the individual, and our current healthcare system may not remain sustainable if we continue to invest in and develop what had served us well in the past five decades. ${ }^{(1)}$

It is intuitive that the answer does not lie in 'more of the same'. An increase in healthcare spending does not always translate to better outcomes due to the complexity of the healthcare situation. The fundamental question that should be asked is what will likely deliver better value or outcome per unit cost in the coming years. The new funding model for healthcare should be one that moves away from the episodic, fee-for-service model to one that is outcome- or value-based for a population. The targeted outcome or value should be patient-centred, with each member of the healthcare system providing for all the needs of a patient as a whole. ${ }^{(9)}$ Members of the healthcare system should no longer be called healthcare 'stakeholders'. This term is passé, and was used when we built and developed our healthcare system in parts.(1) As we move toward a more patientcentric healthcare system, healthcare institutions and services would be more correctly termed 'providers' because they serve as providers for the healthcare needs of a patient. The term 'stakeholders' should be reserved for the public, patients, payers (the government, employers or other third-party payers), policymakers and the healthcare system (the regional health system and their partners).

There are three main areas that we can focus on in order to deliver better value - healthcare integration, strengthening of primary healthcare, and collaboration for health in the community. These are further elaborated in the following text.

\section{HEALTHCARE INTEGRATION}

Healthcare integration is about the development of interfaces, such that each provider in the healthcare system is able to provide for the needs of the patient as a whole. ${ }^{(10-12)}$ The ability of any system to successfully execute an integration depends on purposeful planning and high-level coordination between providers. This involves a vastly different approach to that of developing world-class specialist care. The Eastern Health Alliance's Health Management Unit and Transitional Care department are in-house examples of integrated care. Some ways in which our regional healthcare system is tightening the healthcare network to deliver care for Singaporeans residing in the East include collaborations with the Agency of Integrated Care and Saint Andrew's Community Hospital to develop home care services, as well as with the Salvation Army Peacehaven Nursing Home to set up Grace Corner, so as to provide focused and lower-intensity rehabilitation services for patients who require a longer rehabilitation period. There are other integration initiatives, such as the Post Acute Care @ Home (PACH), Project Care - Advanced Care Planning (ACP) and Virtual Hospital by the National Healthcare Group, which includes Tan Tock Seng Hospital, for the community staying in the central part of Singapore.

\section{STRENGTHENING PRIMARY HEALTHCARE}

Internationally, there has been an emphasis on strengthening primary healthcare, as this has been shown to deliver greater value globally. ${ }^{(2,6,12)}$ The World Health Organization's World Health Report 2008 re-emphasises that a primary healthcare approach is the most efficient, fair and cost-effective way to organise a healthcare system. ${ }^{(2,11-13)}$ It has also been shown to slow down disease progression, reduce disease burden, as well as prevent people with minor complaints from inappropriately using the emergency department (ED) of hospitals. Decades of experience tell us that a focus on primary healthcare produces better outcomes and higher user satisfaction at lower costs. The

${ }^{1}$ SingHealth Polyclinics, ${ }^{2}$ Intergrated Care (Clinical), Eastern Health Alliance, Singapore

Correspondence: Prof Fock Kwong Ming, Assistant Chief Executive Officer, Integrated Care (Clinical), Eastern Health Alliance, 5 Tampines Central 1, Tampines Plaza, \#08-01/05, Singapore 529541. Kwong.Ming.Fock@easternhealth.sg 
Eastern Community Health Centre is a new model of care providing ancillary support to the private primary healthcare sector, and the GPFirst programme encourages the community to visit their family doctor before presenting to the ED, so as to reduce the number of inappropriate presentations to the ED.

The close collaboration between SingHealth Polyclinics (primary healthcare) and Eastern Health Alliance (a regional healthcare system) aims to reshape the primary healthcare landscape and develop early strategies to arrest the trends of selected disease conditions that have a long natural progression, including congestive heart failure, chronic obstructive pulmonary disease, diabetes mellitus, hip fracture in the elderly, stroke, mental health, frail elderly and cancers. Early detection of these chronic diseases and their optimal control are proven strategies. $^{(2-8,10,12,13)}$ Close examination of how a strengthened primary healthcare network, together with other healthcare partners, can provide a seamless transition of care for an individual is crucial to minimise duplications of services and to close any gaps in care delivery within our system.

\section{COLLABORATION FOR HEALTH IN THE COMMUNITY}

The World Health Report 2008 also promotes primary healthcare as a people-centred approach to health that makes prevention as important as cure. ${ }^{(13)}$ As part of the preventive approach to tackle the root causes of ill health, healthcare providers must work with non-traditional partners in the community to offer an upstream attack on threats to health. Early interventions do not address the onset of disease, lifestylerelated risk factors or precursor conditions. To reduce these risk factors, health systems will need to move out of their comfort zones and into the community. ${ }^{(11-13)}$ Arresting individual risks through collaborations with community partners appears to be the key intervention to avoid the premature development of chronic diseases, decrease the need for expensive and complicated treatments in tertiary hospitals, and deliver improved outcomes at a possibly lower overall cost. Such an effort will often require healthcare providers to collaborate with nontraditional partners in the communities to identify, design and execute programmes or interventions. The Eastern Health Alliance's Eastern Community Health Outreach (ECHO) programme is an example of a community outreach effort to residents living in Tampines, a district in the eastern region of Singapore.

\section{CONCLUSION}

Singapore is blessed with a strong foundation in specialties development. Moving forward, the focus will be to work on improving transitions of care between healthcare providers, strengthening our primary healthcare network, and increasing collaborations with partners within the community, so that the healthcare system will be better able to provide for the patient's needs and the population's health.

\section{REFERENCES}

1. Lee HS. Opening Address. World Health Summit Regional Meeting, April 8, 2013, Singapore.

2. OECD iLibrary. Health at a Glance 2013: OECD Indicators [online]. Available at: http://dx.doi.org/10.1787/health_glance-2013-en. Accessed February 28, 2014.

3. Roehrig CS, Rousseau DM. The growth in cost per case explains far more of US health spending increases than rising disease prevalence. Health Aff (Millwood) 2011; 30:1657-63.

4. Gaziano T. Cardiovascular disease in the developing world and its costeffective management. Circulation 2005; 112:3547-53.

5. Gaziano TA, Bitton A, Anand S, Abrahams-Gessel S, Murphy A. Growing epidemic of coronary heart disease in low and middle income countries. Curr Probl Cardiol 2010; 35:72-115.

6. Süssmuth-Dyckerhoff C, Wang J. China's health care reforms. Health International 2010; 10:54-67.

7. Alliance for Aging Research. The Silver Book: Chronic Disease and Medical Innovation in an Aging Nation, 2006.

8. Engberding N, Wenger NK. Cardiovascular disease prevention tailored for women. Expert Rev Cardiovasc Ther 2008; 6:1123-34.

9. Henke N, Kelsey T, Whately H. Transparency: the most powerful driver of health care improvement? Health International 2011; 11:64-73.

10. Jamison DT, Breman JG, Measham AR, et al, eds. Priorities in Health. Disease Control Priorities Project. Washington (DC): World Bank, 2006.

11. Briggs CJ, Garner P. Strategies for integrating primary health services in middle and low-income countries at the point of delivery. Cochrane Database Syst Rev 2006; 19:CD003318.

12. World Health Organization. Everybody's Business: Strengthening Health Systems to Improve Health Outcomes. WHO's Framework for Action. Geneva: WHO, 2007.

13. World Health Organization. The World Health Report 2008: Primary health care (now more than ever). Geneva: WHO, 2008. 